\title{
ON MINIMAL SETS OF SCALAR PARABOLIC EQUATIONS WITH SKEW-PRODUCT STRUCTURES
}

\author{
WENXIAN SHEN AND YINGFEI YI
}

Abstract. Skew-product semi-flow $\Pi_{t}: X \times Y \rightarrow X \times Y$ which is generated by

$$
\left\{\begin{array}{l}
u_{t}=u_{x x}+f\left(y \cdot t, x, u, u_{x}\right), \quad t>0,0<x<1, y \in Y, \\
D \text { or } N \text { boundary conditions }
\end{array}\right.
$$

is considered, where $X$ is an appropriate subspace of $H^{2}(0,1),(Y, \mathbb{R})$ is a minimal flow with compact phase space. It is shown that a minimal set $E \subset X \times Y$ of $\Pi_{t}$ is an almost 1-1 extension of $Y$, that is, set $Y_{0}=$ $\left\{y \in Y \mid \operatorname{card}\left(E \subset P^{-1}(y)\right)=1\right\}$ is a residual subset of $Y$, where $P: X \times Y \rightarrow$ $Y$ is the natural projection. Consequently, if $(Y, \mathbb{R})$ is almost periodic minimal, then any minimal set $E \subset X \times Y$ of $\Pi_{t}$ is an almost automorphic minimal set. It is also proved that dynamics of $\Pi_{t}$ is closed in the category of almost automorphy, that is, a minimal set $E \subset X \times Y$ of $\Pi_{t}$ is almost automorphic minimal if and only if $(Y, \mathbb{R})$ is almost automorphic minimal. Asymptotically almost periodic parabolic equations and certain coupled parabolic systems are discussed. Examples of nonalmost periodic almost automorphic minimal sets are provided.

\section{INTRODUCTION}

In the current paper, we shall study fundamental dynamical issues for a family of scalar parabolic equations

$$
u_{t}=u_{x x}+f\left(y \cdot t, x, u, u_{x}\right), \quad t>0,0<x<1,
$$

with boundary conditions

$$
\beta u(t, 0)+(1-\beta) u_{x}(t, 0)=0, \quad \beta u(t, 1)+(1-\beta) u_{x}(t, 1)=0, \quad t>0,
$$

where $\beta=0$ or $1, y \in Y,(Y, \mathbb{R})$ is a minimal flow with compact metric phase space $Y$, and the function $f(y, x, u, p)$ is $C^{2}$ in $x, u, p$, and Lipschitz in $y$.

Let $X$ be a fractional power space associated with the operator $u \rightarrow-u_{x x}$ : $\mathscr{D} \rightarrow L^{2}(0,1)$ that satisfies $X \hookrightarrow C^{1}[0,1]$, where $\mathscr{D}=\left\{u \mid u \in H^{2}(0,1)\right.$, $u$ satisfies (1.2)\} [21]. It is well known that for any $y \in Y, U \in X$, equation $(1.1)_{y}-(1.2)$ admits a (locally) unique solution $u(t, \cdot, U, y)$ in $X$ with

Received by the editors May 27, 1994 and, in revised form, December 14, 1994.

1991 Mathematics Subject Classification. Primary 35B15, 35K57, 54H20.

Partially supported by NSF grant DMS 9207069. 
$u(0, \cdot, U, y)=U(\cdot)$. Thus equations $(1.1)_{y}-(1.2) \quad(y \in Y)$ generate a (local) skew-product semi-flow $\Pi_{t}$ on $X \times Y$,

$$
\Pi_{t}(U, y)=(u(t, \cdot, U, y), y \cdot t), \quad t>0 .
$$

It is important to note that the (local) skew-product semi-flow (1.3) restricted to an $\omega$-limit set or a minimal invariant set defines a usual skew-product (twosided) flow ([20], [21]).

One of the fundamental dynamical issues in the study of a skew-product flow is the lifting properties of minimal sets (for general discussion, see [15], [33], [35], and references therein). In terms of differential equations, the lifting properties describe whether or how much certain structure (say, periodicity, almost periodicity) in an equation can be lifted to its solutions. A lifting property is also closely related to the asymptotic behavior of bounded solutions of a differential equation (see Section 4 and discussion below). Before stating the main results of the current paper, we first introduce some terminology.

Since only real flows are considered in the current paper, without causing confusion, we shall simply denote a flow $(E, \mathbb{R})$ as $E$. Let $p: E \rightarrow Y$ be a homomorphism of minimal flows (that is, $p: E \rightarrow Y$ is continuous and surjective, and $p(x \cdot t)=p(x) \cdot t$ for any $x \in E, t \in \mathbb{R} ;$ see [39], [40]). Assume that $E$ is a compact metric space. $E$ is a 1-1 extension of $Y$ if card $E \cap p^{-1}(y)=1$ for all $y \in Y$. E is an almost 1-1 extension of $Y$ if there is a residual subset $Y_{0} \subset Y$ such that card $E \cap p^{-1}\left(y_{0}\right)=1$ for $y_{0} \in Y_{0}$. Note that a 1-1 extension of $Y$ is topologically conjugate to $Y$. A 1-1 extension (almost 1-1 extension) of an almost periodic minimal flow $Y$ (see Section 3 ) is referred to as an almost periodic (almost automorphic) extension of $Y$. The notion of almost automorphy was first introduced in dynamical systems by S. Bochner [4]. An almost automorphic function (see Section 3) is the pointwise limit of a 'jointly almost automorphic' net of (Bohr) almost periodic functions [39]. It has many properties in common with the usual (Bohr) almost periodic function, for example, it possesses (nonunique) Fourier series in a deterministic way [39]. An almost periodic function is necessarily almost automorphic, but the converse is not true (see [41]). The connection between the almost automorphy and the almost periodicity is indicated in [39] as follows: a function is (Bohr) almost periodic if and only if its compact hull (with compact open topology) consists of almost automorphic functions. Unlike the almost periodic case, the compact hull of a continuous (nonalmost periodic) almost automorphic function contains only residually many almost automorphic functions (see Section 3 or [39] for details). We refer to the closure of an almost automorphic (almost periodic) motion in a dynamical system as an almost automorphic (almost periodic) minimal set (see Section 3). It is known that a flow $E$ is an almost automorphic minimal flow if and only if it is an almost automorphic extension of an almost periodic minimal flow $Y$, and the almost automorphic points on $E$ (points $x \in E$ such that $x \cdot t$ is almost automorphic) are precisely those $x$ with $E \cap p^{-1}(y)=x$ for some $y \in Y$ (see Section 3 or [39]). Thus, an almost automorphic minimal set contains residually many almost automorphic points (an almost periodic minimal set consists of almost periodic hence almost automorphic points).

In the study of scalar almost periodic equations $(1.1)_{y}-(1.2)$ (that is, $Y$ in $(1.1),-(1.2)$ is almost periodic minimal) as well as almost periodic scalar ODEs (note that scalar ODEs form subdynamical systems of $(1.1)_{y}-(1.2)$ with 
$\beta=0$ ), the existence of almost automorphic solutions is crucial. There are many examples (see [18], [24] and Section 5 of the current paper) in which a scalar almost periodic ODE admits almost automorphic solutions but no almost periodic solutions. In other words, in such systems, there is a minimal set which is almost automorphic but not almost periodic. In the current paper, we shall show that 'almost automorphy' is essential for dynamical system $(1.1)_{y}-(1.2)$ or (1.3). Let $P: X \times Y \rightarrow Y$ be the natural projection. Then for any minimal set $E \subset X \times Y$ of (1.3), $P: E \rightarrow Y$ clearly defines a homomorphism of minimal flows. Our main results are as follows.

Main theorem. (1) Any minimal set $E$ of (1.3) is an almost 1-1 extension of $Y$.

(2) A minimal set $E$ of (1.3) is almost automorphic minimal if and only if $Y$ is almost automorphic minimal.

We conclude from (1) that any minimal set of (1.3) is an almost automorphic extension of $Y$ if $Y$ is almost periodic minimal. This solves a problem left open in [37].

There are two important facts involved in our main results. First, the result (1) is optimal as is suggested by examples in Section 5. Second, by the result (2), although the lifting property is not 'closed' in the category of almost periodicity, it is however 'closed' in the category of almost automorphy.

We would like to point out that there are major differences between the periodic case and the almost periodic case in the study of $(1.1)_{y}-(1.2)$ or (1.3). In the case that the minimal flow $Y$ in $(1.1)_{y}-(1.2)$ is periodic (that is, $Y$ is minimal and any motion in $Y$ is periodic), it is well known that each minimal set of (1.3) is a precise 1-1 extension of $Y$, namely, a periodic minimal set (see [5], [9]). In fact, much more can be said in the case of periodic dependence. For example, each $\omega$-limit set of (1.3) is necessarily a periodic minimal set (a 1-1 extension of $Y$ ), therefore, each positively bounded motion in (1.3) is asymptotically periodic ([5], [9]). Similar results are no longer true in the almost periodic case. It is shown in [37] for the almost periodic case that each $\omega$-limit set of (1.3) may contain two but no more than two minimal sets, moreover, a positively bounded motion $\Pi_{t}\left(U_{0}, y_{0}\right)$ of $(1.3)$ is asymptotically almost periodic if and only if its $\omega$-limit set $\omega\left(U_{0}, y_{0}\right)$ is an almost periodic extension of $Y$ (see [37] or Section 4). Thus, asymptotic almost periodicity cannot be always expected for positively bounded solutions of (1.3). One often observes a kind of 'nonuniform' asymptotic phenomenon in (1.3) if an (nonalmost periodic) almost automorphic motion appears.

To explain this, we note that an alternative way to state the asymptotic almost periodicity is the following: $\Pi_{t}\left(U_{0}, y_{0}\right)$ is asymptotically almost periodic if and only if $\omega\left(U_{0}, y_{0}\right)$ is minimal and every point in $\omega\left(U_{0}, y_{0}\right)$ is an almost automorphic point. In general, if $\omega\left(U_{0}, y_{0}\right)$ is a nonalmost periodic minimal set (hence an almost automorphic extension of $Y$ ), then one cannot conclude that $\Pi_{t}\left(U_{0}, y_{0}\right)$ is asymptotically almost automorphic (this is unlikely to be true even if $\omega\left(U_{0}, y_{0}\right) \cap P^{-1}\left(y_{0}\right)$ is a single almost automorphic point). Instead, $\Pi_{t}\left(U_{0}, y_{0}\right)$ is 'almost' asymptotically almost automorphic if $\omega\left(U_{0}, y_{0}\right)$ is minimal since in this case $\omega\left(U_{0}, y_{0}\right)$ contains residually many almost automorphic points.

The paper is organized as follows. We prove our main result (1) in Section 2 
by using the properties of the zero number developed in [1], [8], [27]. Cases in which a minimal set $E$ is a precise 1-1 extension of $Y$ are also discussed in Section 2. In Section 3, we give our attention to cases in which $Y$ is either an almost automorphic or an almost periodic minimal flow, and prove the main result (2). Some applications of our current work to nonautonomous parabolic equations (for example, almost periodic equations, asymptotically almost periodic equations, and certain recursively coupled systems) are provided in Section 4. In Section 5, we discuss two examples of almost periodic scalar ODEs from Fink [18] and Johnson [24].

We remark that the results in this paper also hold true for equation $(1.1)_{y}$ with more general boundary conditions (e.g. third boundary conditions).

Dynamical studies of scalar parabolic equations in one space dimension go back to [6], [28], [29] for autonomous equations and are developed later in [2], [5], [7], [9], [10], [17], [34] for periodic dependent equations. Almost periodic dependent cases are discussed in [37], [38]. In [38], work of [11], [12] on Floquet theory is applied. For dynamical studies of parabolic equations in higher space dimensions, see [13], [14], [30], [31], [36], [42], [43] and references therein.

Acknowledgment. We would like to thank X.-Y. Chen, R. A. Johnson, K. Lu, J. Mallet-Paret, P. Poláčik for comments and discussions. We are grateful to S.-N. Chow and J. K. Hale for their continuous encouragement and support. We also thank R. Ellis for comments and references.

\section{LIFTING PROPERTIES OF MINIMAL SETS}

The main subject of this section is to study lifting properties for minimal invariant sets of (1.3). We note that any minimal set of (1.3) is compact and the flow $\Pi_{t}$ restricted to it defines a global (two-sided) flow [20].

Definition 2.1. For a given $C^{1}$ function $u:[0,1] \rightarrow \mathbb{R}^{1}$, the zero number $Z(u(\cdot))$ of $u$ is defined as

$$
Z(u(\cdot))=\#\{x \in(0,1) \mid u(x)=0\} .
$$

The following lemma is proved in [8] (see also [1], [27] for cases with stronger conditions).

Lemma 2.1. Consider the following scalar linear parabolic equation:

$$
\left\{\begin{array}{l}
u_{t}=a(t, x) u_{x x}+b(t, x) u_{x}+c(t, x) u, \quad t>0, \quad x \in(0,1), \\
\beta u(t, 0)+(1-\beta) u_{x}(t, 0)=0, \quad \beta u(t, 1)+(1-\beta) u_{x}(t, 1)=0, \quad t>0,
\end{array}\right.
$$

where $a, a_{t}, a_{x}, b$ and $c$ are bounded continuous functions, $a \geq \delta>0$. Let $u(t, x)$ be a classical nontrivial solution of (2.1). Then the following holds:

(1) $Z(u(t, \cdot))$ is finite for $t>0$ and is nonincreasing in $t$;

(2) $Z(u(t, \cdot))$ can drop only at $t_{0}$ such that $u\left(t_{0}, \cdot\right)$ has a multiple zero in $[0,1]$;

(3) $Z(u(t, \cdot))$ can drop only finite many times, and there exists a $t^{*}>0$ such that $u(t, \cdot)$ has only simple zeros in $[0,1]$ as $t \geq t^{*}($ hence $Z(u(t, \cdot))=$ constant as $\left.t \geq t^{*}\right)$.

Our next lemma can also be found in [37] or [40]. 
Lemma 2.2. Let $p: E \rightarrow Y$ be homomorphism of minimal flows, where $E, Y$ are compact $T_{2}$. Then there is a residual subset $Y_{0} \subset Y$ which is invariant and satisfies the following properties:

For any $y_{*} \in Y_{0}, y \in Y$ and any $x_{*} \in p^{-1}\left(y_{*}\right)$, if $\left\{t_{n}\right\}$ is a sequence with $t_{n} \rightarrow+\infty$ or $-\infty$ such that $y \cdot t_{n} \rightarrow y_{*}$ as $n \rightarrow \infty$, then there is a sequence $\left\{x_{n}\right\} \subset p^{-1}(y)$ such that $x_{n} \cdot t_{n} \rightarrow x_{*}$ as $n \rightarrow \infty$.

Proof. Consider the set-valued map $q: Y \rightarrow 2^{E}, y \mapsto p^{-1}(y)$, where $2^{E}$ is furnished with Hausdorff metric $\sigma$. Recall, for any $E_{1}, E_{2} \in 2^{E}, \sigma\left(E_{1}, E_{2}\right)=$ $\max \left\{\mu\left(E_{1}, E_{2}\right), \mu\left(E_{2}, E_{1}\right)\right\}$, where $\mu\left(E_{1}, E_{2}\right)=\max _{x \in E_{1}} \min _{x^{\prime} \in E_{2}} d_{E}\left(x, x^{\prime}\right)$, $d_{E}$ is the metric on $E$. It is easy to see that $q$ is upper semicontinuous. Let $Y_{0} \subset Y$ be the set of continuous points of $q$. Then $Y_{0} \subset Y$ is a residual subset [3]. $Y_{0}$ is clearly invariant. Now, the lemma follows immediately from the definition of lower semicontinuity for set-valued maps [3].

Definition 2.2. Let $X, Y$ be as in (1.3) and let $P: X \times Y \rightarrow Y$ be the natural projection. For any $y \in Y$, we define relation ' $\geq$ ' (' $>$ ') on $P^{-1}(y)$ as follows. For any $\left(U_{1}, y\right),\left(U_{2}, y\right) \in P^{-1}(y),\left(U_{1}, y\right) \geq(>)\left(U_{2}, y\right)$ if there is $T>0$ such that

$$
\begin{aligned}
& (1-\beta) u\left(t, 0, U_{1}, y\right)+\beta u_{x}\left(t, 0, U_{1}, y\right) \\
& \quad \geq(>)(1-\beta) u\left(t, 0, U_{2}, y\right)+\beta u_{x}\left(t, 0, U_{2}, y\right)
\end{aligned}
$$

for all $t \geq T$.

Lemma 2.3. For any $y \in Y, \geq$ ' defines a total ordering on $P^{-1}(y)$, i.e. ' $\geq$ ' is a partial ordering of $P^{-1}(y)$ and any $\left(U_{1}, y\right),\left(U_{2}, y\right) \in P^{-1}(y)$ are comparable. Proof. For any $y \in Y$ and $\left(U_{1}, y\right),\left(U_{2}, y\right) \in P^{-1}(y)$, we note that $V(t, x, y)$ $=u\left(t, x, U_{1}, y\right)-u\left(t, x, U_{2}, y\right)$ satisfies a linear parabolic equation of form (2.1) ([5], [37]). Therefore, by Lemma 2.1, if $U_{1} \neq U_{2}$, then there is a $T>0$ such that

$(1-\beta)\left(u\left(t, 0, U_{1}, y\right)-u\left(t, 0, U_{2}, y\right)\right)+\beta\left(u_{x}\left(t, 0, U_{1}, y\right)-u_{x}\left(t, 0, U_{2}, y\right)\right)$

has constant sign for $t \geq T$. The rest of the proof is straightforward.

Definition 2.3. For any $y \in Y$, let $A(y) \subset P^{-1}(y)$ be a subset such that card $A(y) \geq 2$. For any $\left(U_{1}, y\right),\left(U_{2}, y\right) \in A(y), U_{1} \neq U_{2}$, define

$$
Z_{+}\left(U_{1}, U_{2}, y\right)=\min _{t \geq 0} Z\left(u\left(t, \cdot, U_{1}, y\right)-u\left(t, \cdot, U_{2}, y\right)\right),
$$

and

$$
N_{0}(A(y))=\min \left\{Z_{+}\left(U_{1}, U_{2}, y\right) \mid\left(U_{1}, y\right),\left(U_{2}, y\right) \in A(y), U_{1} \neq U_{2}\right\} .
$$

For any $\left(U_{0}, y\right) \in A(y)$, define

$$
A\left(U_{0}, y\right)=\left\{(U, y) \in A(y) \mid U \neq U_{0}, Z_{+}\left(U, U_{0}, y\right)=N_{0}(A(y))\right\} .
$$

The above quantities are well defined by Lemma 2.1 .

Lemma 2.4. Let $E \subset X \times Y$ be a minimal set of (1.3), $Y_{0} \subset Y$ be the residual subset in Lemma 2.2, and $A(y)=E \cap P^{-1}(y) \quad(y \in Y)$. If $y \in Y_{0}$ is such that 
card $A(y) \geq 2$, then $A\left(U_{0}, y\right)$ defined in (2.4) is nonempty for any $\left(U_{0}, y\right) \in$ $A(y)$.

Proof. Take $y \in Y_{0}$ so that $\operatorname{card} A(y) \geq 2$. Let $\left(U_{1}^{*}, y\right),\left(U_{2}^{*}, y\right) \in A(y)$, $U_{1}^{*} \neq U_{2}^{*}$ be such that $Z_{+}\left(U_{1}^{*}, U_{2}^{*}, y\right)=N_{0}(A(y))$. Choose a $T>0$ such that $u\left(t, \cdot, U_{1}^{*}, y\right)-u\left(t, \cdot, U_{2}^{*}, y\right)$ has only simple zeros on $[0,1]$ as $t \geq T$. This implies that $Z\left(u\left(t, \cdot, U_{1}^{*}, y\right)-u\left(t, \cdot, U_{2}^{*}, y\right)\right)=N_{0}(A(y))$ for $t \geq T$. Now for any $\left(U_{0}, y\right) \in A(y)$, by Lemma 2.2, there are sequences $t_{n} \rightarrow \infty$ and $\left\{\left(U_{n}, y\right)\right\} \subset A(y)$ such that $y \cdot t_{n} \rightarrow y, \Pi_{t_{n}}\left(U_{0}, y\right) \rightarrow\left(U_{1}^{*}, y\right)$, and $\Pi_{t_{n}}\left(U_{n}, y\right)$ $\rightarrow\left(U_{2}^{*}, y\right)$ as $n \rightarrow \infty$. It follows that there exists $n_{0}>0$ such that $Z\left(u\left(t_{n}+T, \cdot, U_{0}, y\right)-u\left(t_{n}+T, \cdot, U_{n}, y\right)\right)=N_{0}(A(y))$ for $n \geq n_{0}$. Since $N_{0}(A(y))$ is the smallest zero number on $A(y)$, one has that $Z_{+}\left(U_{0}, U_{n}, y\right)=$ $N_{0}(A(y))$ for $n \geq n_{0}$. Clearly, $U_{n} \neq U_{0}$ for $n \geq n_{0}$. This proves the lemma.

Lemma 2.5. Let $E, Y_{0}, A(y)$ be as in Lemma 2.4. Then for any $y \in Y_{0}$ with card $A(y) \geq 2$, there is a $\left(U_{0}, y\right) \in A(y)$ such that $\left(U_{0}, y\right) \geq(U, y)$ for any $(U, y) \in A\left(U_{0}, y\right)$.

Proof. Suppose that the lemma is not true. Then there is a $y^{*} \in Y_{0}$ with card $A\left(y^{*}\right) \geq 2$, and, for any $\left(U^{*}, y^{*}\right) \in A\left(y^{*}\right)$, there is a $\left(U_{0}^{*}, y^{*}\right) \in A\left(U^{*}, y\right)$ such that $\left(U^{*}, y^{*}\right)<\left(U_{0}^{*}, y^{*}\right)$. Since $Z_{+}\left(U_{0}^{*}, U^{*}, y^{*}\right)=N_{0}\left(A\left(y^{*}\right)\right)$, by Lemma 2.1 , there is a $T>0$ such that $u\left(t, \cdot, U_{0}^{*}, y\right)-u\left(t, \cdot, U^{*}, y^{*}\right)$ has only simple zeros on $[0,1]$ and $Z\left(u\left(t, \cdot, U_{0}^{*}, y^{*}\right)-u\left(t, \cdot, U^{*}, y^{*}\right)\right)=N_{0}\left(A\left(y^{*}\right)\right)$ for all $t \geq T$. In particular, $u\left(T, \cdot, U_{0}^{*}, y^{*}\right)-u\left(T, \cdot, U^{*}, y^{*}\right)$ has only simple zeros on $[0,1]$. It follows that there is an open neighborhood $B\left(U^{*}, y^{*}\right)$ of $\left(U^{*}, y^{*}\right)$ in $A\left(y^{*}\right)$ such that $u\left(T, \cdot, U_{0}^{*}, y^{*}\right)-u\left(T, \cdot, U, y^{*}\right)$ has only simple zeros on $[0,1]$ and $Z\left(u\left(T, \cdot, U_{0}^{*}, y^{*}\right)-u\left(T, \cdot, U, y^{*}\right)\right)=N_{0}(A(y))$ for any $\left(U, y^{*}\right) \in B\left(U^{*}, y^{*}\right)$. Without loss of generality, we also assume that

$$
\begin{aligned}
& (1-\beta)\left|u\left(T, 0, U^{*}, y^{*}\right)-u\left(T, 0, U, y^{*}\right)\right| \\
& +\beta\left|u_{x}\left(T, 0, U^{*}, y^{*}\right)-u_{x}\left(T, 0, U, y^{*}\right)\right| \\
& <\frac{1}{2}\left[(1-\beta)\left(u\left(T, 0, U_{0}^{*}, y^{*}\right)-u\left(T, 0, U^{*}, y^{*}\right)\right)\right. \\
& \left.\quad+\beta\left(u_{x}\left(T, 0, U_{0}^{*}, y^{*}\right)-u_{x}\left(T, 0, U^{*}, y^{*}\right)\right)\right]
\end{aligned}
$$

for any $\left(U, y^{*}\right) \in B\left(U^{*}, y^{*}\right)$. This implies

$$
\begin{aligned}
(1-\beta) & \left(u\left(T, 0, U_{0}^{*}, y^{*}\right)-u\left(T, 0, U, y^{*}\right)\right) \\
+ & \beta\left(u_{x}\left(T, 0, U_{0}^{*}, y^{*}\right)-u_{x}\left(T, 0, U, y^{*}\right)\right)>0
\end{aligned}
$$

for any $\left(U, y^{*}\right) \in B\left(U^{*}, y^{*}\right)$. Since $N_{0}\left(A\left(y^{*}\right)\right)$ is the smallest zero number on $A\left(y^{*}\right)$, it is clear that $u\left(t, \cdot, U_{0}^{*}, y^{*}\right)-u\left(t, \cdot, U, y^{*}\right)$ has only simple zeros on $[0,1]$, and

$$
Z\left(u\left(t, \cdot, U_{0}^{*}, y^{*}\right)-u\left(t, \cdot, U, y^{*}\right)\right)=N_{0}\left(A\left(y^{*}\right)\right)
$$

for any $t \geq T$ and $\left(U, y^{*}\right) \in B\left(U^{*}, y^{*}\right)$. Combining the above with (2.6), one has that $\left(U, y^{*}\right)<\left(U_{0}^{*}, y^{*}\right)$ for any $\left(U, y^{*}\right) \in B\left(U^{*}, y^{*}\right)$, that is, $B\left(U^{*}, y^{*}\right)$ has an upper bound $\left(U_{0}^{*}, y^{*}\right)$ in $A\left(y^{*}\right)$. Note that $\left\{B\left(U^{*}, y^{*}\right):\left(U^{*}, y^{*}\right) \in\right.$ $\left.A\left(y^{*}\right)\right\}$ forms an open covering of the compact set $A\left(y^{*}\right)$. Hence there are 
$\left(U_{i}^{*}, y^{*}\right) \in A\left(y^{*}\right), i=1,2, \ldots, n_{0}$, for some $n_{0} \geq 1$, such that

$$
\bigcup_{i=1}^{n_{0}} B\left(U_{i}^{*}, y^{*}\right)=A\left(y^{*}\right) \text {. }
$$

Let $\left(U_{0}, y^{*}\right) \in A\left(y^{*}\right)$ be the maximum element of the chain

$$
\left\{\left(U_{01}^{*}, y^{*}\right), \ldots,\left(U_{0 n_{0}}^{*}, y^{*}\right)\right\}
$$

(i.e. $\left.\left(U_{0}, y^{*}\right) \geq\left(U_{0 i}^{*}, y^{*}\right), i=1,2, \ldots, n_{0}\right)$, where $\left(U_{0 i}^{*}, y^{*}\right) \in A\left(y^{*}\right), i=$ $1,2, \ldots, n_{0}$, are upper bounds of $B\left(U_{i}^{*}, y^{*}\right)$. Then $\left(U_{0}, y^{*}\right)$ is a maximum element of $A\left(y^{*}\right)$. Now, by Lemma $2.4, A\left(U_{0}, y^{*}\right)$ is nonempty. Clearly, $\left(U_{0}, y^{*}\right)$ is also an upper bound of $A\left(U_{0}, y^{*}\right)$, that is, for any $\left(U, y^{*}\right) \in$ $A\left(U_{0}, y^{*}\right),\left(U_{0}, y^{*}\right)>\left(U, y^{*}\right)$. This is a contradiction.

Theorem 2.6. Any minimal set $E$ of (1.3) is an almost 1-1 extension of $Y$, that is, there is a residual set $Y_{0} \subset Y$ such that $\operatorname{card}\left(E \cap P^{-1}(y)\right)=1$ for all $y \in Y_{0}$. Proof. Let $E \subset X \times Y$ be a minimal set of (1.3) and $Y_{0} \subset Y$ the residual set in Lemma 2.2. Suppose there is a $y \in Y_{0}$ such that $\operatorname{card}\left(E \cap P^{-1}(y)\right)>1$. By Lemma 2.5 , there is a $\left(U_{0}, y\right) \in A(y) \equiv E \cap P^{-1}(y)$ such that $\left(U_{0}, y\right) \geq(U, y)$ for all $(U, y) \in A\left(U_{0}, y\right)$. Recall that

$$
A\left(U_{0}, y\right)=\left\{(U, y) \in E \mid Z_{+}\left(U_{0}, U, y\right)=N_{0}(A(y))\right\},
$$

and

$$
N_{0}(A(y))=\min \left\{Z_{+}\left(U_{1}, U_{2}, y\right) \mid\left(U_{1}, y\right),\left(U_{2}, y\right) \in A(y), U_{1} \neq U_{1}\right\} .
$$

Take $\left(U_{1}^{*}, y\right),\left(U_{2}^{*}, y\right) \in A(y)$, with $U_{1}^{*} \neq U_{2}^{*}$, such that $Z_{+}\left(U_{1}^{*}, U_{2}^{*}, y\right)=$ $N_{0}(A(y))$. By Lemma 2.1 and the invariance of $Y_{0}$, without loss of generality, we assume that $u\left(t, \cdot, U_{1}^{*}, y\right)-u\left(t, \cdot, U_{2}^{*}, y\right)$ has only simple zeros in $[0,1]$ for $t \geq 0$, and $\left(U_{1}^{*}, y\right)>\left(U_{2}^{*}, y\right)$. By minimality of $E$ and Lemma 2.2 , there are sequences $t_{n} \rightarrow \infty$ and $\left\{\left(U_{n}, y\right)\right\} \subset A(y)$ such that $\Pi_{t_{n}}\left(U_{0}, y\right) \rightarrow\left(U_{2}^{*}, y\right)$, $\Pi_{t_{n}}\left(U_{n}, y\right) \rightarrow\left(U_{1}^{*}, y\right)$ as $n \rightarrow \infty$. Similar to the arguments in Lemma 2.4 and Lemma 2.5, there is an $r_{0}>0$ such that $u\left(t_{n}, \cdot, U_{0}, y\right)-u\left(t_{n}, \cdot, U_{n}, y\right)$ has only simple zeros on $[0,1]$, and $Z_{+}\left(U_{0}, U_{n}, y\right)=N_{0}(A(y))$ for $n \geq$ $n_{0}$. Thus, $u\left(t, \cdot, U_{0}, y\right)-u\left(t, \cdot, U_{n}, y\right)$ has only simple zeros on $[0,1]$, and $Z\left(u\left(t, \cdot, U_{0}, y\right)-u\left(t, \cdot, U_{n}, y\right)\right)=N_{0}(A(y))$ for $t \geq t_{n}, n \geq n_{0}$. This implies that

$$
(1-\beta)\left(u\left(t, 0, U_{0}, y\right)-u\left(t, 0, U_{n}, y\right)\right)+\beta\left(u_{x}\left(t, 0, U_{0}, y\right)-u_{x}(t, 0, U, y)\right)
$$

has constant sign for $t \geq t_{n}, n \geq n_{0}$. By choosing a larger $n_{0}$ if necessary, one has that $(1-\beta)\left(u\left(t_{n}, 0, U_{0}, y\right)-u\left(t_{n}, 0, U_{n}, y\right)\right)+\beta\left(u_{x}\left(t_{n}, 0, U_{0}, y\right)-\right.$ $\left.u_{x}\left(t_{n}, 0, U_{n}, y\right)\right)$ has the same sign as $(1-\beta)\left(U_{2}^{*}(0)-U_{1}^{*}(0)\right)+\beta\left(U_{2 x}^{*}(0)-\right.$ $\left.U_{1 x}^{*}(0)\right)$ for $n \geq n_{0}$. Since $\left(U_{2}^{*}, y\right)<\left(U_{1}^{*}, y\right)$, it follows from the above discussions that $\left(U_{0}, y\right)<\left(U_{n}, y\right)$ for $n \geq n_{0}$. Note that $\left\{\left(U_{n}, y\right)\right\} \subset A\left(U_{0}, y\right)$ and $\left(U_{n}, y\right) \leq\left(U_{0}, y\right)$ for $n \geq n_{0}$. We then have a contradiction.

Definition 2.4. Given a flow $E$ with compact metric phase space $(E, d)$, points $x_{1}, x_{2} \in E$ are said to be proximal if

$$
\inf _{t \in \mathbb{R}} d\left(x_{1} \cdot t, x_{2} \cdot t\right)=0 .
$$


A point $x \in E$ which is proximal only to itself is said to be distal. $E$ is called a point-distal flow if there is a distal point $x \in X$ such that its orbit $\{x \cdot t \mid t \in \mathbb{R}\}$ is dense in $E . E$ is distal flow if every point in $E$ is distal.

Let $p: E \rightarrow Y$ be a homomorphism of minimal flows. A point $x \in p^{-1}(y)$ is a fibre distal point if $x$ is not proximal with any point in $p^{-1}(y) \backslash\{x\} . E$ is a fibre distal flow if any point in $E$ is fibre distal.

Remark 2.1. (1) A point-distal flow is necessarily minimal ([15], [40]). Distal flow laminates into minimal sets [15].

(2) Let $E \subset X \times Y$ be a minimal invariant set of (1.3). By Theorem 2.6, if $y \in$ $Y$ is such that card $E \cap P^{-1}(y)>1$, then any two points $\left(U_{1}, y\right),\left(U_{2}, y\right) \in E \cap$ $P^{-1}(y)$ are proximal. To see this, let $\left(U_{0}, y_{0}\right)$ be a point such that $\left(U_{0}, y_{0}\right)=$ $E \cap P^{-1}\left(y_{0}\right)$. Then there is a sequence $t_{n} \rightarrow \infty$ such that $y \cdot t_{n} \rightarrow y_{0}$ as $n \rightarrow \infty$. Take a subsequence if necessary; one sees that $\Pi_{t_{n}}\left(U_{i}, y\right) \rightarrow\left(U_{0}, y_{0}\right)$ $(i=1,2)$ as $n \rightarrow \infty$. Thus, a point $(U, y)$ in a minimal set $E$ of $(1.3)$ is a fibre distal point if and only if $(U, y)=E \cap P^{-1}(y)$.

Corollary 2.7. Let $E \subset X \times Y$ be a minimal set of (1.3). Then $E$ is point-distal if and only if $Y$ is point-distal.

Proof. The 'only if' part is obvious since the projection $P: E \rightarrow Y$ is a flow homomorphism. We now prove the 'if' part. By Theorem 2.6, there is a residual subset $Y_{0} \subset Y$ such that $\operatorname{card}\left(E \cap P^{-1}(y)\right)=1$ for $y \in Y_{0}$. Let $Y_{1}$ be the set of distal points of $Y . Y_{1}$ is a residual subset of $Y$ [16]. Hence $Y^{*}=Y_{0} \cap Y_{1} \subset Y$ is also residual. Now let $y \in Y^{*}$ and $(U, y)=E \cap P^{-1}(y)$. If $\left(U^{*}, y^{*}\right) \in E$ is proximal to $(U, y)$, then $y^{*}, y$ are proximal. It follows that $y^{*}=y$. Since card $E \cap P^{-1}(y)=1,\left(U^{*}, y^{*}\right)=(U, y)$. Above all, for any $y \in Y^{*}$, $(U, y)=E \cap P^{-1}(y)$ is a distal point of $E$.

Corollary 2.8. Let $Y$ in (1.3) be a distal flow. Then any minimal set $E$ of (1.3) is point-distal. Moreover, $(U, y) \in E$ is a distal point if and only if $(U, y)=E \cap P^{-1}(y)$.

Proof. Note that in the case $Y$ is distal, a point in $E$ is a fibre distal point if and only if it is a distal point. The corollary then follows immediately from Corollary 2.7 and Remark 2.1 (2).

We now discuss cases in which a minimal set $E$ of (1.3) can be a precise 1-1 extension of $Y$.

Definition 2.5. A motion $\Pi_{t}\left(U_{0}, y_{0}\right)$ of $(1.3)$ is said to be uniformly stable if, for any $\varepsilon>0$, there is $\delta=\delta(\varepsilon)>0$ such that if

$$
\left\|u\left(\tau, \cdot, U_{0}, y_{0}\right)-u\left(\tau, \cdot, U_{1}, y_{0}\right)\right\|<\delta(\varepsilon)
$$

for some $\left(U_{1}, y_{0}\right) \in X \times Y$, and some $\tau \in \mathbb{R}^{+}$, then

$$
\left\|u\left(t+\tau, \cdot, U_{0}, y_{0}\right)-u\left(t+\tau, \cdot, U_{1}, y_{0}\right)\right\|<\varepsilon
$$

for all $t \in \mathbb{R}^{+}$.

Theorem 2.9. A minimal set $E$ of (1.3) is a 1-1 extension of $Y$ provided one of the following conditions holds.

(1) $E$ is fibre distal; 
(2) There is a $\left(U_{0}, y_{0}\right) \in E$ such that $\Pi_{t}\left(U_{0}, y_{0}\right)$ is uniformly stable;

(3) $E$ is hyperbolic (that is, the linearized equation of the flow on $E$ admits an exponential dichotomy over $E$, see [38] for details);

(4) Equations (1.1) $)_{y}-(1.2)$ are monotone in the sense that $f_{u}(y, x, u, p) \leq 0$ for all $(y, x, u, p) \in Y \times[0,1] \times \mathbb{R}^{1} \times \mathbb{R}^{1}$.

Proof. (1) follows from Remark 2.1 (2).

(2) By arguments in [33] or [35], the uniformly stability implies the fibre distality.

(3) This is proved in [38] in the case that $Y$ is an almost periodic minimal set. The proof in [38] can be carried over for general minimal set $Y$.

(4) By applying the strong maximum principle ([19], [32]) for parabolic equations, one shows that the monotonicity implies that each minimal set of (1.3) is fibre distal (see [37] for details).

Remark 2.2. The statement (3) in Theorem 2.9 is in fact true for any $\omega$-limit set, that is, if an $\omega$-limit set $\omega\left(U_{0}, y_{0}\right)$ is hyperbolic, then it is a precise 1-1 extension of $Y$ (see [38]).

We end this section by stating a structure theorem of the $\omega$-limit set $\omega\left(U_{0}, y_{0}\right)$ for a positively bounded motion $\Pi_{t}\left(U_{0}, y_{0}\right)=\left(u\left(t, \cdot, U_{0}, y_{0}\right), y_{0} \cdot t\right)$ of $(1.3)$, that is, $u\left(t, \cdot, U_{0}, y_{0}\right)(t \geq 0)$ is bounded in $X$. Following [21] and the standard a priori estimates for parabolic equations, we know that if $u\left(t, \cdot, U_{0}, y_{0}\right)$ $\left(U_{0} \in X\right)$ is bounded in $X$ for $t$ in the existence interval of the solution, then $u$ is a globally defined classical solution; moreover, for any $\delta>0$, $\{u(t, x, U, g) \mid t \geq \delta\}$ is relatively compact both in $X$ and in $H^{2}(0,1)$. Therefore $\omega\left(U_{0}, y_{0}\right)$ is a nonempty connected compact subset of $X \times Y$ [20].

Theorem 2.10. Consider (1.3). Let $\left(U_{0}, y_{0}\right) \in X \times Y$ be such that the motion $\Pi_{t}\left(U_{0}, y_{0}\right)(t>0)$ is bounded. Then the $\omega$-limit set $\omega\left(U_{0}, y_{0}\right)$ contains at most two minimal sets. More precisely, one of the following is true:

(1) $\omega\left(U_{0}, y_{0}\right)=E_{1} \cup E_{2} \cup E_{12}$, where $E_{1}, E_{2}$ are minimal sets, $E_{12} \neq \varnothing . E_{12}$ connects $E_{1}, E_{2}$ in the sense that if $\left(U_{12}, y\right) \in E_{12}$, then $\omega\left(U_{12}, y\right) \cap\left(E_{1} \cup E_{2}\right) \neq$ $\varnothing, \alpha\left(U_{12}, y\right) \cap\left(E_{1} \cup E_{2}\right) \neq \varnothing$ (here $\alpha$ is referred to as the $\alpha$-limit set $)$.

(2) $\omega\left(U_{0}, y_{0}\right)=E_{1} \cup E_{11}$, where $E_{1}$ is minimal, $E_{11} \neq \varnothing, E_{11}$ connects $E_{1}$ in the sense that if $\left(U_{11}, y\right) \in E_{11}$, then $\omega\left(U_{11}, y\right) \cap E_{1} \neq \varnothing, \alpha\left(U_{11}, y\right) \cap E_{1} \neq$ $\varnothing$.

(3) $\omega\left(U_{0}, y_{0}\right)$ is a minimal invariant set.

Theorem 2.11. Any two minimal invariant sets $E_{1}, E_{2}$ of (1.3) are separated in the following sense:

(1) $\left[a_{1}(y), b_{1}(y)\right] \cap\left[a_{2}(y), b_{2}(y)\right]=\varnothing$ for all $y \in Y$, where

$$
\begin{aligned}
& a_{i}(y)=\min \left\{(1-\beta) U(0)+\beta U_{x}(0) \mid(U, y) \in E_{i} \cap P^{-1}(y)\right\}, \\
& b_{i}(y)=\max \left\{(1-\beta) U(0)+\beta U_{x}(0) \mid(U, y) \in E_{i} \cap P^{-1}(y)\right\}
\end{aligned}
$$

$(i=1,2)$.

If $a_{1}\left(y^{*}\right)-b_{2}\left(y^{*}\right)>0$ for some $y^{*} \in Y$, then there is $a \delta>0$ such that $a_{1}(y)-b_{2}(y) \geq \delta$ for all $y \in Y$.

The proof of Theorem 2.10 and Theorem 2.11 are almost identical to those in the almost periodic case [37]. 
Remark 2.3. There is an example in the almost periodic case in which an $\omega$ limit set contains precisely two minimal sets (see [37]).

\section{Almost AUTOMORPHIC AND ALMOST PERIODIC EXTENSIONS}

In this section, we give our particular attention to cases in which flow $Y$ in (1.3) is either an almost automorphic or an almost periodic minimal flow.

Definition 3.1. (1) Let $E$ be a complete metric space. A continuous function $f: \mathbb{R}^{1} \rightarrow E$ is said to be almost periodic if from every sequence $\left\{t_{n}^{\prime}\right\}$ one can extract a subsequence $\left\{t_{n}\right\} \subset \mathbb{R}^{1}$ such that $\lim _{n \rightarrow \infty} f\left(t+t_{n}\right)$ exists uniformly in $t \in \mathbb{R}^{1} . f$ is said to be almost automorphic if $\left\{t_{n}\right\} \subset \mathbb{R}^{1}$ and $g: \mathbb{R}^{1} \rightarrow E$ are such that $f\left(t_{n}+t\right) \rightarrow g(t)$ as $n \rightarrow \infty$ pointwise in $t \in \mathbb{R}^{1}$, then $g\left(t-t_{n}\right) \rightarrow f(t)$ as $n \rightarrow \infty$ pointwise.

(2) Let $E$ be a flow with compact metric phase space $E$. A point $x \in E$ is called an almost periodic (almost automorphic) point if its orbit $x \cdot t$ is an almost periodic (almost automorphic) function of $t$. The flow $E$ is called almost periodic (almost automorphic) minimal if it contains an almost periodic (almost automorphic) point $x_{0} \in E$ such that the orbit $\left\{x_{0} \cdot t\right\}$ is dense in $E$.

Remark 3.1. (1) By definition, an almost periodic point (function) is necessarily an almost automorphic point (function) ([18], [26]). We shall see in Lemma 3.1 that an almost automorphic point $x_{0}$ is necessarily a distal point in a dynamical system. It then follows from Remark 2.1 that $\operatorname{cl}\left\{x_{0} \cdot t\right\}$ must be minimal.

(2) It is well known that the flow is almost periodic minimal if and only if it is equicontinuous ([26], [35]). Moreover, any almost periodic minimal flow is distal [15].

Definition 3.2. Consider a homomorphism of minimal flows $p: E \rightarrow Y$ with $Y$ being almost periodic minimal. $E$ is an (almost automorphic) almost periodic extension of $Y$ if $E$ is an (almost) 1-1 extension of $Y$.

Remark 3.2. Let $E, Y, p$ be as above.

(1) If $E$ is an almost automorphic extension of $Y$, then $E$ is an almost automorphic minimal set since it is clear that the set $\left\{x \in p^{-1}(y) \mid \operatorname{card}^{-1}(y)=\right.$ $1, y \in Y\}$ consists of almost automorphic points.

(2) If $E$ is an almost periodic extension of $Y$, then $E$ is an almost periodic minimal set since $p$ in this case is an isomorphism, and $E=p^{-1}(Y)$ is equicontinuous (Remark $3.1(2))$.

Lemma 3.1. Let $p: E \rightarrow Y$ be a homomorphism of minimal flows and assume that $E$ is a compact metric space, $Y$ is almost periodic minimal. If $E$ is an almost automorphic extension of $Y$, then $\{x \in E \mid x$ is an almost automorphic point $\}=\{x \in E \mid x$ is a distal point $\}=\left\{x \in E \mid \operatorname{card} p^{-1}(p x)=1\right\}=\{x \in$ $p^{-1}\left(y_{0}\right) \mid q: y \rightarrow p^{-1}(y)$ is continuous at $y_{0} \in Y$ with the Hausdorff metric on $\left.2^{E}\right\}$.

Proof. Let $Y_{0} \subset Y$ be the residual set satisfying properties of Lemma 2.2, i.e. $Y_{0}=\left\{y \in Y \mid q: Y \rightarrow 2^{E}, y \mapsto p^{-1}(y)\right.$ is continuous at $y_{0}$ in the Hausdorff metric of $\left.2^{E}\right\}$. Since $E$ is an almost automorphic extension of $Y$, by Lemma 2.2 , it is easy to see that $Y_{0}=\left\{y \in Y \mid \operatorname{card} p^{-1}(y)=1\right\}$. Adopting arguments from Corollary 2.7 and Corollary 2.8, one has that $Y_{0}=\left\{y \in Y \mid x=p^{-1}(y)\right.$ is distal $\}$. Let $Y^{*}=\left\{y \in Y \mid\right.$ there is an almost automorphic point $\left.x \in p^{-1}(y)\right\}$. 
Then $Y_{0} \subset Y^{*}(\operatorname{Remark} 3.2(1))$. Now, if $y \in Y$ is such that there is an almost automorphic point $x \in p^{-1}(y)$, then we claim that $\operatorname{card} p^{-1}(y)=1$. If not, then there is a $x^{*} \in p^{-1}(y)$ such that $x^{*} \neq x$. Fix a $y_{0} \in Y_{0}$ and denote $x_{0}=p^{-1}\left(y_{0}\right)$. Let $\left\{t_{n}\right\}$ be a sequence such that $x_{0} \cdot t_{n} \rightarrow x^{*}$ as $n \rightarrow \infty$. Since $x \cdot\left(-t_{n}\right) \rightarrow x_{0}$, it follows that $x_{0} \cdot t_{n} \rightarrow x$ as $n \rightarrow \infty$, that is, $x^{*}=x$. This is a contradiction.

Corollary 3.2. (1) $Y$ is almost automorphic minimal if and only if it contains residually many almost automorphic points.

(2) $Y$ is almost periodic minimal if and only if it consists of almost automorphic points.

Proof. (1) By [39], $Y$ is almost automorphic minimal if and only if it is an almost automorphic extension of an almost periodic minimal flow $\bar{Y}$. Note that an almost automorphic minimal flow is point-distal and a point-distal flow consists of residually many distal points [16]. (1) then follows from Lemma 3.1 .

(2) The proof is a straightforward application of Lemma 3.1 and the above part (1). We only note that an almost periodic minimal flow is distal. A more general situation is discussed in [39].

Theorem 3.3. Let $E \subset X \times Y$ be a minimal set of (1.3). Then $E$ is almost automorphic if and only if $Y$ is almost automorphic.

Proof. If $E$ is almost automorphic, then $Y$ is clearly almost automorphic. We now assume that $Y$ is almost automorphic. By Theorem 2.6 and Corollary 3.2, the set $Y^{*}=\left\{y \in Y \mid y\right.$ is almost automorphic, card $\left.E \cap P^{-1}(y)=1\right\}$ is a residual subset of $Y$. Now take $y \in Y^{*}$ and denote $(U, y)=E \cap P^{-1}(y)$. Let $\left\{t_{n}\right\}$ be a sequence such that $\Pi_{t_{n}}(U, y)$ converges to some $\left(U^{*}, y^{*}\right) \in E$ as $n \rightarrow \infty$. Since $y^{*} \cdot\left(-t_{n}\right) \rightarrow y$ as $n \rightarrow \infty$ and card $E \cap P^{-1}(y)=1$, it follows that $\Pi_{-t_{n}}\left(U^{*}, y^{*}\right) \rightarrow(U, y)$ as $n \rightarrow \infty$. This shows that $(U, y)$ is an almost automorphic point.

Theorem 3.4. Let $Y$ in (1.3) be almost periodic minimal. Then the following holds.

(1) Any minimal set $E \subset X \times Y$ of (1.3) is an almost automorphic extension of $Y$.

(2) A minimal set $E \subset X \times Y$ of (1.3) is an almost periodic extension of $Y$ provided that one of the conditions in Theorem 2.9 holds.

(3) If $E \subset X \times Y$ of (1.3) is almost periodic minimal, them it is an almost periodic extension of $Y$.

Proof. (1) is a corollary of Theorem 2.6.

(2) follows from Theorem 2.9 .

(3) is a consequence of Lemma 3.1, Corollary 3.2, and (1) of the current theorem.

Remark 3.3. Theorems 3.3 and 3.4 are 'sharp' in the following sense.

(1) Theorem 3.3 implies that dynamics of (1.3) is 'closed' in the category of almost automorphy. Nevertheless, as shown in Section 5 (see also [24], [25], [37]), dynamics of (1.3) is not 'closed' in the category of almost periodicity. 
That is, even $Y$ is almost periodic minimal, a minimal set $E$ of (1.3) can be nonalmost periodic almost automorphic.

(2) Dynamics of (1.3) is however 'closed' for periodicity since any almost 1-1 extension of a periodic minimal set $Y$ is a precise 1-1 extension of $Y$ by the periodicity. In fact, in this case, it is shown in [5], [9] that any $\omega$-limit set of (1.3) is a precise $1-1$ extension of $Y$, that is, a periodic minimal set with the same period as that of $Y$.

Remark 3.4. Consider a family of scalar ODEs:

$$
u^{\prime}=f(y \cdot t, u), \quad u \in \mathbb{R}^{1}, y \in Y,
$$

where $Y$ is a compact minimal flow. Equations $(3.1)_{y}$ generate a skew-product flow $\Pi_{t}$ on $\mathbb{R}^{1} \times Y$,

$$
\Pi_{t}\left(u_{0}, y\right)=\left(u\left(t, u_{0}, y\right), y \cdot t\right),
$$

where $u\left(t, u_{0}, y\right)$ is the solution of $(3.1)_{y}$ with $u\left(0, u_{0}, y\right)=u_{0}$.

Let the function $f$ in $(1.1)_{y}$ be independent of $x, u_{x}$, and let $\beta=0$ in (1.2). Then (3.2) defines a subflow of (1.3). Thus, all our results so far hold true for (3.2) with $X=\mathbb{R}^{1}$.

\section{Applications}

Let us consider a scalar parabolic equation of the following form: (4.1)

$\left\{\begin{array}{l}u_{t}=u_{x x}+F\left(t, x, u, u_{x}\right), \quad t>0,0<x<1, \\ \beta u(t, 0)+(1-\beta) u_{x}(t, 0)=0, \quad \beta u(t, 1)+(1-\beta) u_{x}(t, 1)=0, \quad t>0,\end{array}\right.$

where $\beta=0$ or $1, F$ is $C^{2}$, and for every compact set $K \subset[0,1] \times \mathbb{R}^{1} \times$ $\mathbb{R}^{1}, F(t, x, u, p)$ with all its partial derivatives up to order 2 are uniformly continuous and bounded on $\mathbb{R}^{1} \times K$. We shall show that (4.1) with all its 'translated' and 'limiting' equations together generates a skew-product semi-flow of form $(1.1)_{y}-(1.2)$, or (1.3). The construction is based on ideas of classical topological dynamical system (see, for example, [35]).

Standard Hypothesis. Consider a $C^{2}$ function $F: \mathbb{R}^{1} \times[0,1] \times \mathbb{R}^{m} \rightarrow \mathbb{R}^{n} . F$ is said to be standard if, for any compact subset $K \subset[0,1] \times \mathbb{R}^{m}, F$ and all its partial derivatives up to order 2 are uniformly continuous and bounded on $\mathbb{R}^{1} \times K$.

Proposition 4.1 ([22], [23], [45]). Consider a function $F$ which satisfies the standard hypothesis. Then there exists a Lipschitz flow $Y_{0}$ (i.e., $(y, t)=y \cdot t$ is Lipschitz in both $y$ and $t$ ) with $Y_{0}=H(F)$ (the hull of $f$ ) being a compact metric space, and a function $f: Y_{0} \times[0,1] \times \mathbb{R}^{m} \rightarrow \mathbb{R}^{n}$ such that the following holds:

(1) $f: Y_{0} \times[0,1] \times \mathbb{R}^{m} \rightarrow \mathbb{R}^{n},(y, x, z) \mapsto f(y, x, z)$ is $C^{2}$ in $x, z$ and Lipschitz in $y$;

(2) There is a $y_{0} \in Y_{0}$ such that

$$
f\left(y_{0} \cdot t, x, z\right) \equiv F(t, x, z) .
$$

Proof. Let $C$ be the space of continuous functions $S: \mathbb{R}^{1} \times[0,1] \times \mathbb{R}^{m} \rightarrow \mathbb{R}^{n}$ with the compact open topology. This topology is metrizable. To be more 
precise, for $S_{1}, S_{2} \in C$, define a metric $d$ as follows,

$$
d\left(S_{1}, S_{2}\right) \doteq \sum_{n=1}^{\infty} \frac{\min \left\{1,\left\|S_{1}-S_{2}\right\|_{n}\right\}}{2^{n}},
$$

where $\left\|S_{1}-S_{2}\right\|_{n}=\sup _{|t| \leq n, 0 \leq x \leq 1,|z| \leq n}\left|S_{1}(t, x, z)-S_{2}(t, x, z)\right|$. It is easy to see that the translation $(S, t) \mapsto S_{t}, S_{t}(s, x, z)=S(s+t, x, z)$ defines a flow on $(C, d)$ [35] and $Y_{0}=H(F)=\operatorname{cl}\left\{F_{t} \mid t \in \mathbb{R}^{1}\right\}$, the hull of $F$, is compact (Ascoli's Theorem), and translation invariant.

Define $f: Y_{0} \times[0,1] \times \mathbb{R}^{m} \rightarrow \mathbb{R}^{n},(y, x, z) \mapsto y(0, x, z)$ and let $y_{0}=F$. Then $f\left(y_{0} \cdot t, x, z\right)=y_{0} \cdot t(0, x, z)=y_{0}(t, x, z)=F(t, x, z)$. For other properties of $Y_{0}$ and $f$, see [22], [23], [45] for more details.

We note that $f$ needs only to be Lipschitz in $t$ for the above proposition to holds.

Proposition 4.2. Let $F: \mathbb{R}^{1} \times[0,1] \times \mathbb{R}^{m} \rightarrow \mathbb{R}^{n}$ be a function satisfying the standard hypothesis and let $Y_{0}=H(F)$ be as in Lemma 4.1. Then the following holds.

(1) If, for each $(x, z) \in[0,1] \times \mathbb{R}^{m}, F(\cdot, x, z): \mathbb{R}^{1} \rightarrow \mathbb{R}^{m}$ is an almost automorphic function, then $Y_{0}$ is an almost automorphic minimal flow.

(2) If, for each $(x, z) \in[0,1] \times \mathbb{R}^{m}, F(\cdot, x, z): \mathbb{R}^{1} \rightarrow \mathbb{R}^{m}$ is an almost periodic function, then $Y_{0}$ is an almost periodic minimal flow.

Proof. (1) We only observe that $y_{0}=F$ is an almost automorphic point of $Y_{0}$.

(2) is a standard fact (see [35]).

Now, consider (4.1) and let $f$ be the function corresponding to $F$ in Proposition 4.1. Then (4.1) gives rise to a family of equations over $Y_{0}=H(F)$ :

$(4.2)_{y}$

$\begin{cases}u_{t}=u_{x x}+f\left(y \cdot t, x, u, u_{x}\right), & t>0,0<x<1, \\ \beta u(t, 0)+(1-\beta) u_{x}(t, 0)=0, & \beta u(t, 1)+(1-\beta) u_{x}(t, 1)=0, \quad t>0,\end{cases}$ where $\beta=0$ or $1, y \in Y_{0}$. Note that $f(y \cdot t, x, u, p)=y(t, x, u, p)$ and if $y_{0}=F$, then (4.2) $y_{0}$ coincides with (4.1). Equations (4.2)y $\left(y \in Y_{0}\right)$ then generate a (local) skew-product semi-flow $\Pi_{t}$ on $X \times Y_{0}$ (here $X$ is as in (1.3)):

$$
\Pi_{t}\left(u_{0}, y\right)=\left(u\left(t, \cdot, u_{0}, y\right), y \cdot t\right) \text {. }
$$

Since $Y_{0}$ is compact invariant, it contains a minimal set $Y$. All results in Section 2 therefore apply to the (local) skew-product semi-flow $\Pi_{t}$ restricted to $X \times Y$.

4.1. Asymptotic behavior of positively bounded solutions.

We now discuss asymptotic behavior of positively bounded solutions of (4.1).

Definition 4.1. Consider a positively bounded solution $u(t, x)$ of $(4.1)$, that is, $\{u(t, \cdot) \mid t \geq 0\}$ is bounded in $X . u(t, \cdot)$ is said to be asymptotically almost periodic if there is an almost periodic function $u^{*}: \mathbb{R}^{1} \rightarrow X, t \mapsto u^{*}(t, \cdot)$, such that $\left\|u(t, \cdot)-u^{*}(t, \cdot)\right\| \rightarrow 0$ as $t \rightarrow \infty$.

Proposition 4.3. Consider (4.1) and assume that the function $F$ satisfies the condition (2) of Proposition 4.2. Let $u(t, \cdot)$ be a positively bounded solution of (4.1). Then $u(t, \cdot)$ is asymptotically almost periodic if and only if the $\omega$-limit 
set $\omega\left(U_{0}, F\right)$ in (4.3) is an almost periodic extension of $H(F)$ (here $U_{0}(\cdot)=$ $u(0, \cdot))$.

Proof. Suppose $u(t, \cdot)$ is asymptotically almost periodic; i.e., $\left\|u(t, \cdot)-u^{*}(t, \cdot)\right\|$ $\rightarrow 0$ as $t \rightarrow \infty$ for some almost periodic function $u^{*}: \mathbb{R}^{1} \rightarrow X, t \mapsto u^{*}(t, \cdot)$. Let $t_{n} \rightarrow \infty$ be such that $\Pi_{t_{n}}\left(U_{0}, F\right) \rightarrow(\tilde{U}, F) \in \omega\left(U_{0}, F\right)$ and $u^{*}\left(t+t_{n}, \cdot\right) \rightarrow$ $u^{*}(t, \cdot) \quad\left(t \in \mathbb{R}^{1}\right)$ as $n \rightarrow \infty$. Then

$$
\left\|u(t, \cdot, \tilde{U}, F)-u^{*}(t, \cdot)\right\|=\lim _{n \rightarrow \infty}\left\|u\left(t+t_{n}, \cdot\right)-u^{*}\left(t+t_{n}, \cdot\right)\right\|=0 .
$$

Hence $u^{*}(t, \cdot)$ is an almost periodic solution of $(4.1)$ (or $\left.(4.2)_{F}\right)$ and $\omega\left(U_{0}, F\right)$ $=\operatorname{cl}\left\{\Pi_{t}\left(U^{*}, F\right) \mid t \in \mathbb{R}^{1}\right\}$, where $U^{*}=u^{*}(0, \cdot)$. Therefore, $\omega\left(U_{0}, F\right)$ is almost periodic minimal. By Theorem 3.4(3), $\omega\left(U_{0}, F\right)$ is an almost periodic extension of $H(F)$.

Conversely, suppose that $\omega\left(U_{0}, F\right)$ is an almost periodic extension of $H(F)$. Let $\left(U^{*}, F\right)=\omega\left(U_{0}, F\right) \cap P^{-1}(F)$. Then $u^{*}\left(t, \cdot, U^{*}, F\right)$ is an almost periodic solution of $(4.2)_{F}$. Now, one must have $\left\|u(t, \cdot)-u^{*}\left(t, \cdot, U^{*}, F\right)\right\| \rightarrow 0$ as $t \rightarrow$ $\infty$ (hence $u(t, \cdot)$ is asymptotically almost periodic). For otherwise, there is a sequence $t_{n} \rightarrow \infty$ such that $\Pi_{t_{n}}\left(U_{0}, F\right) \rightarrow\left(\widetilde{U}_{0}, \widetilde{F}\right)$ and $\Pi_{t_{n}}\left(U^{*}, F\right) \rightarrow\left(\widetilde{U}^{*}, \widetilde{F}\right)$ as $n \rightarrow \infty$, where $\widetilde{U}_{0} \neq \widetilde{U}^{*}$. Obviously, $\left(\widetilde{U}_{0}, \widetilde{F}\right),\left(\widetilde{U}^{*}, \widetilde{F}\right) \in \omega\left(U_{0}, F\right)$. This implies that $\omega\left(U_{0}, F\right)$ is not a 1-1 extension of $H(F)$ or $\omega\left(U_{0}, F\right)$ is not an almost periodic extension of $H(F)$. This is a contradiction.

For a positively bounded solution $u(t, x)$ of $(4.1)$, denote $U_{0}(\cdot)=u(0, \cdot)$. By Proposition $4.3, u(t, \cdot)$ is asymptotically almost periodic if and only if $\omega\left(U_{0}, F\right)$ is minimal and consists of almost automorphic points. Now let us assume for the moment that $\omega\left(U_{0}, F\right)$ is minimal but not almost periodic. By Theorem 3.4, $\omega\left(U_{0}, F\right)$ contains only residually many almost automorphic points, and each almost automorphic point $\left(U^{*}, y^{*}\right)$ corresponds to an almost automorphic solution $u\left(t, x, U^{*}, y^{*}\right)$ of $(4.2)_{y^{*}}$. Comparing with the asymptotic almost periodicity, one then concludes that $u(t, \cdot)$ is 'almost' asymptotically almost automorphic. Note that even if $\omega\left(U_{0}, F\right) \cap P^{-1}(F)$ is a single almost automorphic point, $u(t, x)$ may not be asymptotically almost automorphic in general.

4.2. Asymptotically almost automorphic or asymptotically almost periodic equations.

Let $F$ in (4.1) be either asymptotically almost automorphic or asymptotically almost periodic, that is, $F=F_{0}+h$, where $F_{0}$ satisfies condition (1) or (2) in Proposition 4.2, $h \rightarrow 0$ as $t \rightarrow \infty$ uniformly on compact set $K \subset[0,1] \times \mathbb{R}^{2}$.

In this case, if $u_{0} \in X$ is such that the solution $u\left(t, \cdot, u_{0}, F\right)$ of (4.1) is bounded in $X$ for $t>0$, then the $\omega$-limit set $\omega\left(u_{0}, F\right) \subset X \times Y$ is compact invariant under flow $\Pi_{t}: X \times Y \rightarrow X \times Y$, where $Y=H\left(F_{0}\right)$ is either an almost automorphic or an almost periodic minimal set. By Theorem 3.3, $\omega\left(u_{0}, F\right)$ contains an almost automorphic minimal set $E$. Let $\left(u^{*}, y^{*}\right)$ be an almost automorphic point in $E$. Then there is a sequence $\left\{t_{n}\right\}$ with $t_{n} \rightarrow \infty$ as $n \rightarrow \infty$ such that $u\left(t+t_{n}, \cdot, u_{0}, F\right) \rightarrow u\left(t, \cdot, u^{*}, y^{*}\right)$ as $n \rightarrow \infty$. Note that $u\left(t, x, u^{*}, y^{*}\right)$ is an almost automorphic solution of (4.2) .* with $u\left(0, x, u^{*}, y^{*}\right)=u^{*}(x)$. If $\omega\left(u_{0}, F\right)$ is minimal and $\left(u^{*}, F_{0}\right) \in$ $\omega\left(u_{0}, F\right)$ is an almost automorphic point, then for 'almost all' sequences $t_{n} \rightarrow$ 
$\infty, u\left(t_{n}, \cdot, u_{0}, F\right)-u\left(t_{n}, \cdot, u^{*}, F_{0}\right) \rightarrow 0$ as $n \rightarrow \infty$. Roughly speaking, $u\left(t, \cdot, u_{0}, F\right)$ is 'almost' asymptotically almost automorphic. If $Y=H\left(F_{0}\right)$ is almost periodic minimal, similar to Proposition 4.3, we have

Proposition 4.4. Suppose that $Y=H\left(F_{0}\right)$ is almost periodic minimal and $u\left(t, \cdot, u_{0}, F\right)$ is a positively bounded solution of (4.1). Then $u\left(t, \cdot, u_{0}, F\right)$ is asymptotically almost periodic if and only if $\omega\left(u_{0}, F\right)$ is an almost periodic extension of $H\left(F_{0}\right)$.

Proof. We use similar arguments as in Proposition 4.3. In fact, if $u\left(t, \cdot, u_{0}, F\right)$ is asymptotically almost periodic, that is, $u\left(t, \cdot, u_{0}, F\right)-u^{*}(t, \cdot) \rightarrow 0$ as $t \rightarrow \infty$ for some almost periodic function $u^{*}(t, \cdot)$, then we can show that $u^{*}(t, \cdot)$ is an almost periodic solution of $(4.2)_{F_{0}}$ and $\omega\left(u_{0}, F\right)=\operatorname{cl}\left\{\Pi_{t}\left(U^{*}, F_{0}\right) \mid t \in \mathbb{R}^{1}\right\}$, where $U^{*}(\cdot)=u^{*}(0, \cdot)$. Hence $\omega\left(u_{0}, F\right)$ is an almost periodic extension of $H\left(F_{0}\right)$. Conversely, if $\omega\left(u_{0}, F\right)$ is an almost periodic extension of $H\left(F_{0}\right)$, let $\left(u^{*}, F_{0}\right)=\omega\left(u_{0}, F\right) \cap P^{-1}\left(F_{0}\right)$. Then $u\left(t, \cdot, u^{*}, F_{0}\right)$ is an almost periodic solution of $(4.2)_{F_{0}}$ and $\left\|u\left(t, \cdot, u_{0}, F\right)-u\left(t, \cdot, u^{*}, F_{0}\right)\right\| \rightarrow 0$ as $t \rightarrow \infty$.

\subsection{System of recursively coupled scalar equations.}

We now look at a system of recursively coupled scalar equations of the following form:

$$
\left\{\begin{array}{r}
u_{t}^{k}=u_{x x}^{k}+F^{k}\left(t, x, u^{k}, u_{x}^{k}, u^{k-1}, u_{x}^{k-1}, \ldots, u^{1}, u_{x}^{1}\right), \\
t>0,0<x<1, \\
\beta^{k} u^{k}(t, 0)+\left(1-\beta^{k}\right) u_{x}^{k}(t, 0)=0, \quad \beta^{k} u^{k}(t, 1)+\left(1-\beta^{k}\right) u_{x}^{k}(t, 1)=0, \\
t>0,
\end{array}\right.
$$

where $\beta^{k}=0$ or $1, k=1,2, \ldots, n, F^{k}(1 \leq k \leq n)$ satisfies the condition (2) of Proposition 4.2. By Proposition 4.1 and Proposition 4.2, there are functions $f^{k} \quad(1 \leq k \leq n)$ and an almost periodic minimal flow $Y$ such that

$$
\begin{aligned}
& f^{k}\left(y_{0} \cdot t, x, u^{k}, p^{k}, u^{k-1}, p^{k-1}, \cdot, u^{1}, p^{1}\right) \\
& \quad \equiv F^{k}\left(t, x, u^{k}, p^{k}, u^{k-1}, p^{k-1}, \cdot, u^{1}, p^{1}\right) \quad(1 \leq k \leq n),
\end{aligned}
$$

here $y_{0}=\left(F^{1}, F^{2}, \ldots, F^{n}\right)^{\top}$ and $Y=H\left(y_{0}\right)$. Then $(4.4)_{k} \quad(1 \leq k \leq n)$ gives rise to equations over $Y$ :

$$
\left\{\begin{array}{r}
u_{t}^{k}=u_{x x}^{k}+f^{k}\left(y \cdot t, x, u^{k}, u_{x}^{k}, u^{k-1}, u_{x}^{k-1}, \ldots, u^{1}, u_{x}^{1}\right), \\
t>0,0<x<1, \\
\beta^{k} u^{k}(t, 0)+\left(1-\beta^{k}\right) u_{x}^{k}(t, 0)=0, \quad \beta^{k} u^{k}(t, 1)+\left(1-\beta^{k}\right) u_{x}^{k}(t, 1)=0, \\
t>0,
\end{array}\right.
$$

where $1 \leq k \leq n, y \in Y$. For each $k$ with $1 \leq k \leq n$, choose a fractional power space $X^{k}$ as in Section 1. Given $k_{0}$ with $1 \leq k_{0} \leq n$, system $(4.5)_{k, y}$, $1 \leq k \leq k_{0}, y \in Y$, then generates a (local) skew-product semi-flow $\Pi_{t}^{k_{0}}$ on $X\left(k_{0}\right) \times Y$, where $X\left(k_{0}\right)=\prod_{k=1}^{k_{0}} X^{k}$,

$$
\begin{aligned}
\Pi_{t}^{k_{0}}\left(u_{0}^{k_{0}}, u_{0}^{k_{0}-1}, \ldots, u_{0}^{1}, y\right) & \\
= & \left(u^{k_{0}}\left(t, \cdot, u_{0}^{k_{0}}, \ldots, u_{0}^{1}, y\right), \ldots,\right. \\
& \left.\left.u^{2}\left(t, \cdot, u_{0}^{2}, u_{0}^{1}, y\right), u^{1}\left(t, \cdot, u_{0}^{1}, y\right)\right), y \cdot t\right),
\end{aligned}
$$


where $u^{k}\left(t, x, u_{0}^{k}, \ldots, u_{0}^{1}, y\right)$ is the solution of $(4.5)_{k, y}$ with

$$
u^{k}\left(0, \cdot, u_{0}^{k}, \ldots, u_{0}^{1}, y\right)=u_{0}^{k}(\cdot) \quad\left(1 \leq k \leq k_{0}\right) .
$$

Observe that, for each $1<k_{0} \leq n, \Pi_{t}^{k_{0}}$ is also a (local) skew-product semiflow on $X^{k_{0}} \times\left(X\left(k_{0}-1\right) \times Y\right)$, that is,

$(4.7)_{k_{0}} \Pi_{t}^{k_{0}}\left(u_{0}^{k_{0}}, U_{0}^{k_{0}-1}, y\right)=\left(u^{k_{0}}\left(t, \cdot, y, u_{0}^{k_{0}}, U_{0}^{k_{0}-1}, y\right), \Pi_{t}^{k_{0}-1}\left(U_{0}^{k_{0}-1}, y\right)\right)$,

where $u_{0}^{k_{0}} \in X^{k_{0}}, U_{0}^{k_{0}-1}=\left(u_{0}^{k_{0}-1}, \ldots, u_{0}^{1}\right)^{\top} \in X\left(k_{0}-1\right)$. Let $X^{*}=X(n)$ and $\Pi_{t}=\Pi_{t}^{n}$. Then $(4.6)_{n}$ or $(4.7)_{n}$ is the (local) skew-product semi-flow on $X^{*} \times Y$ generated by $(4.5)_{k, y}, 1 \leq k \leq n, y \in Y$.

Proposition 4.5. Consider the (local) skew-product semi-flow $\Pi_{t}$ on $X^{*} \times Y$. The following holds.

(1) Any minimal set $X \subset X^{*} \times Y$ of $\Pi_{t}$ is an almost automorphic extension of $Y$.

(2) If $F_{u^{k}}^{k} \leq 0$ for $k=1,2, \ldots, n$, then any minimal set $E \subset X^{*} \times Y$ of $\Pi_{t}$ is an almost periodic extension of $Y$.

Proof. We prove the theorem by induction. For $k=1$, the theorem follows from Theorem 3.4.

Suppose that the theorem holds for some $k_{0}\left(1 \leq k_{0}<n\right)$. Consider $(4.7)_{k_{0}+1}$. Let $E^{k_{0}+1} \subset X\left(k_{0}+1\right) \times Y$ be a minimal set of $\Pi_{t}^{k_{0}+1}$ and let $P^{k_{0}+1}$ : $X\left(k_{0}+1\right) \times Y \rightarrow X\left(k_{0}\right) \times Y$ be the natural projection. Then $P^{k_{0}+1}\left(E^{k_{0}+1}\right) \equiv$ $E^{k_{0}} \subset X\left(k_{0}\right) \times Y$ is a minimal set of $\Pi_{t}^{k_{0}}$. Restricting $\Pi_{t}^{k_{0}+1}$ to $X^{k_{0}+1} \times E^{k_{0}}$ and applying Theorem 2.6, one has that $E^{k_{0}+1}$ is an almost 1-1 extension of $E^{k_{0}}$. Since an almost 1-1 extension of an almost automorphic extension of $Y$ is also an almost automorphic extension of $Y$ (see arguments in Theorem 3.3), (1) is proved.

To prove (2), note that if the condition (2) is satisfied, then, by Theorem 2.9, $E^{k_{0}+1}$ is a precisely $1-1$ extension of $E^{k_{0}}$ which is an almost periodic extension of $Y$.

\section{EXAMPLES}

In this section, we describe two examples from [18] and [24] in which a scalar almost periodic ODE admits a nonalmost periodic almost automorphic solution.

The idea is to construct a certain almost periodic scalar ODE:

$$
x^{\prime}=F(t, x)
$$

which admits no almost periodic solution but a bounded solution. By Lemma $4.1,(5.1)$ generates a family of almost periodic scalar equations on $Y=H(F)$ :

$$
x^{\prime}=f(y \cdot t, x),
$$

where $f: Y \times \mathbb{R}^{1} \rightarrow \mathbb{R}^{1}$ is a function such that $f\left(y_{0} \cdot t, x\right) \equiv F(t, x)$ for $y_{0}=F$. If (5.1) has no almost periodic solution, then the skew-product flow $\Pi_{t}: \mathbb{R}^{1} \times Y \rightarrow \mathbb{R}^{1} \times Y$,

$$
\Pi_{t}\left(x_{0}, y\right)=\left(x\left(t, x_{0}, y\right), y \cdot t\right),
$$


admits no almost periodic point. Let $x\left(t, x_{0}\right)$ be a bounded solution of $(5.1)$ and consider $\omega\left(x_{0}, y_{0}\right)$. By Remark 3.4 and Theorem 3.3, there is an almost automorphic point $\left(x^{*}, y^{*}\right)$ in $\omega\left(x_{0}, y_{0}\right)$. Now, $x\left(t, x^{*}\right)$ is a nonalmost periodic almost automorphic solution to the almost periodic scalar equation $(5.2) y^{*}$.

Example 1 (Johnson [24]). A linear almost periodic scalar ordinary differential equation of form

$$
x^{\prime}+A(t) x=B(t)
$$

is constructed in [24] as follows.

By using so-called Conley-Miller functions, sequences $A_{n}(t), B_{n}(t)$ of periodic functions can be constructed with the following properties:

(1) $A_{n}(t), B_{n}(t)$ are $2^{n}$-periodic $(n=1,2, \ldots)$ and are uniformly convergent. Let $A(t)=\lim _{n \rightarrow \infty} A_{n}(t), B(t)=\lim _{n \rightarrow \infty} B_{n}(t)$. Then $A(t), B(t)$ are almost periodic, $\int_{0}^{t} A(s) d s \rightarrow \infty$ as $t \rightarrow \infty$ and $\bmod B \subset \bmod A$.

(2) For each $n$, the solution $x_{n}(t)$ of the scalar equation

$$
x^{\prime}+A_{n}(t) x=B_{n}(t)
$$

with initial value 0 is $2^{n}$-periodic,

$$
x_{n}\left(2^{n}\right)= \begin{cases}\frac{1}{5}, & n \geq 4, n \text { odd }, \\ 0, & n \text { even }\end{cases}
$$

and $\left|x_{n}(t)\right| \leq 1$.

It is clear that $x_{0}(t)=\lim _{n \rightarrow \infty} x_{n}(t)$ is a bounded solution of (5.4). Now, if (5.4) has an almost periodic solution $\bar{x}(t)$, then $\bmod \bar{x} \subset \bmod A$ [18]. Since $\lim _{n \rightarrow \infty} A\left(2^{n}\right)$ exists, $\lim _{n \rightarrow \infty} \bar{x}\left(2^{n}\right)$ exists [18]. Let $y(t)=\bar{x}(t)-x_{0}(t)$. Then $y(t)$ satisfies

$$
y^{\prime}+A(t) y=0
$$

Thus $y(t)=\bar{x}(0) e^{-\int_{0}^{t} A(s) d s} \rightarrow 0$ as $t \rightarrow \infty$. In particular, $\lim _{k \rightarrow \infty} \bar{x}\left(2^{2 k}\right)=$ $\lim _{k \rightarrow \infty} x_{0}\left(2^{2 k}\right)=0$ and $\lim _{k \rightarrow \infty} \bar{x}\left(2^{2 k+1}\right)=\lim _{k \rightarrow \infty} x_{0}\left(2^{2 k+1}\right)=\frac{1}{5}$. Thus, $\lim _{n \rightarrow \infty} \bar{x}\left(2^{n}\right)$ does not exist, a contradiction.

Example 2 (Fink [18]). Consider a differential equation on the torus

$$
x^{\prime}=f(t, x)
$$

where $f(t+1, x)=f(t, x+1)=f(t, x)$. Let $x(t, \eta)$ be the solution of (5.7) with $x(0, \eta)=\eta$. Consider the Poincaré map $\psi, \eta \mapsto x(1, \eta)$. It is well known that when the rotation number $\rho$ of $\psi$ is irrational, the limit set $S^{\prime}$ of $\left\{\psi^{n}(\eta) \bmod 1, n=1,2, \ldots\right\}$ is either $[0,1]$ or is a cantor set (see [18]).

Now, let $f$ in (5.7) be such that $S^{\prime}$ is a cantor set. Consider

$$
x^{\prime}=f(x+\rho t, t)-\rho .
$$

Clearly, (5.8) is quasi-periodic dependent on $t$ with frequencies 1 and $\rho$. It is shown by Fink [18, Example 12.5] that equation (5.8) admits a bounded solution but admits no almost periodic solution. 


\section{REFERENCES}

1. S. B. Angenent, The zero set of a solution of a parabolic equation, J. Reine Angew. Math. 390 (1988).

2. S. Angenent and B. Fiedler, The dynamics of rotating waves in scalar reaction diffusion equations, Trans. Amer. Math. Soc. 307 (1988).

3. J. P. Aubin and I. Ekeland, Applied nonlinear analysis, Wiley, New York, 1984.

4. S. Bochner, A new approach to almost periodicity, Proc. Nat. Acad. Sci. U.S.A. 48 (1962).

5. P. Brunovský, P. Poláčik and B. Sandstede, Convergence in general periodic parabolic equations in one space dimension, Nonlinear Analysis, Theory, Method and Applications 18 (1992).

6. N. Chafee, Asymptotic behavior for solutions of a one-dimensional parabolic equation with homogeneous Neumann boundary conditions, J. Differential Equations 18 (1975).

7. M. Chen, X. Y. Chen and J. K. Hale, Structural stability for time-periodic one-dimensional parabolic equations, J. Differential Equations 96 (1992).

8. X.-Y. Chen, A strong unique continuation theorem for parabolic equations, (submitted).

9. X.-Y. Chen and H. Matano, Convergence, asymptotic periodicity, and finite-point blow-up in one-dimensional semilinear heat equations, J. Differential Equations 78 (1989).

10. X. Y. Chen and P. Poláčik, Gradient-like structure and Morse decompositions for timeperiodic one-dimensional parabolic equations, Preprint (1993).

11. S.-N. Chow, K. Lu and J. Mallet-Paret, Floquet theory for parabolic differential equations. I: The time periodic case, Preprint.

12. __ Floquet bundles for scalar parabolic equations, Preprint.

13. E. N. Dancer, On the existence of two-dimensional invariant tori for scalar parabolic equations with periodic coefficients, Preprint.

14. E. N. Dancer and P. Hess, Stable subharmonic solutions in periodic reaction-diffusion equations, Preprint (1992).

15. R. Ellis, Lectures on topological dynamics, Benjamin, New York, 1969.

16. _ The Veech's structure theorem, Trans. Amer. Math. Soc. 186 (1973).

17. B. Fiedler and J. Mallet-Paret, A Poincaré-Bendixson theorem for scalar reaction diffusion equations, Arch. Rational Mech. Anal. 107 (1989).

18. A. M. Fink, Almost periodic differential equations, Lecture Notes in Math., vol. 377, SpringerVerlag, Berlin, Heidelberg, and New York, 1974.

19. A. Friedman, Partial differential equations of parabolic type, Prentice-Hall, Englewood Cliffs, NJ, 1964.

20. J. K. Hale, Asymptotic behavior of dissipative systems, Math. Surveys Monographs, Amer. Math. Soc., Providence, RI, 1988.

21. D. Henry, Geometric theory of semilinear parabolic equations, Lecture Notes in Math., vol. 840, Springer-Verlag, Berlin, 1981.

22. R. A. Johnson, Concerning a theorem of Sell, J. Differential Equations 30 (1978).

23. __ On a Floquet theory for almost periodic two-dimensional linear systems, J. Differential Equations 37 (1980).

24. Math. Soc. 82 (1981).

25. __ On almost-periodic linear differential systems of Millionščikov and Vinograd, J. Math. Anal. Appl. 85 (1982).

26. B. M. Levitan and V. V. Zhikov, Almost periodic functions and differential equations, Cambridge Univ. Press, Cambridge and New York, 1982.

27. H. Matano, Nonincrease of the lap-number of a solution for a one-dimensional semilinear parabolic equation, J. Fac. Sci. Univ. Tokyo Sect. IA 29 (1982).

28. Convergence of solutions of one-dimensional semilinear parabolic equations, J. Math. Kyoto Univ. 18-2 (1978) 
29. Asymptotic behavior of solutions of semilinear heat equations on the circle (W. M. $\mathrm{Ni}$, L. A. Peletier, J. Serrin, eds.), Nonlinear Diffusion Equations and Their Equilibrium States, Springer-Verlag, New York, 1986.

30. P. Poláčik, Convergence in smooth strongly monotone flows defined by semilinear parabolic equations, J. Differential Equations 79 (1989).

31. __ Complicated dynamics in scalar semilinear parabolic equations in higher space dimension, J. Differential Equations 89 (1991).

32. M. H. Protter and H. F. Weinberger, Maximum principles of equations, Prentice-Hall, Englewood Cliffs, NJ, 1967.

33. R. J. Sacker and G. R. Sell, Lifting properties in skew-product flows with applications to differential equations, Mem. Amer. Math. Soc. 11 (1977).

34. B. Sandstede and B. Fiedler, Dynamics of periodically forced parabolic equations on the circle, Ergodic Theory Dynamical Systems 12 (1992).

35. G. R. Sell, Topological dynamics and ordinary differential equations, Van Nostrand-Reinhold, 1971.

36. K. Schmitt and J. R. Ward, Jr., Periodic and almost periodic solutions of nonlinear evolution equations, Preprint.

37. W. Shen and Y. Yi, Dynamics of almost periodic scalar parabolic equations, J. Differential Equations (to appear).

38. _ Asymptotic almost periodicity of scalar parabolic equations with almost periodic time dependence, J. Differential Equations (to appear).

39. W. A. Veech, Almost automorphic functions on groups, Amer. J. Math. 87 (1965).

40. _ Point-distal flows, Amer. J. Math. 92 (1970).

41. __ Almost automorphic functions, Proc. Nat. Acad. Sci. U.S.A. 49 (1963).

42. P. Vuillermot, Almost-periodic attractors for a class of nonautonomous reaction-diffusion equations on $\mathbb{R}^{N}$, I. Global stabilization process, J. Differential Equations 94 (1991).

43. __ Almost-periodic attractors for a class of nonautonomous reaction-diffusion equations on $\mathbb{R}^{N}$, II. Codimension-one stable manifolds, Preprint; III. Center curves and Liapunov stability, Preprint.

44. J. R. Ward, Jr., Bounded and almost periodic solutions of semi-linear parabolic equations, Rocky Mountain J. Math. 18 (1988).

45. Y. Yi, A generalized integral manifold theorem, J. Differential Equations 102 (1993).

Department of Mathematics, Auburn University, Auburn University, Alabama 36849

E-mail address: ws@math. auburn.edu

School of Mathematics, Georgia Institute of Technology, Atlanta, Georgia 30332

E-mail address: yi@math.gatech.edu 